\title{
Niobium Films for Superconducting Accelerating Cavities
}

\author{
C. Benvenuti, N. Circelli, M. Hauer
}

\section{IEP/IIBRARY}

\section{ABSTRACT}

Superconducting accelerating cavities made of Nb-coated copper were produced. A maximum acceleration field of $8.6 \mathrm{Mvm}^{-1}$ was reached without quench thanks to the large thermal conductivity of copper at liquid He temperatures.

\section{Introduction}

In the present plans for the Large Electron Positron Collider (LEP), the construction of which has begun at CERN, it is foreseen to upgrade the initial electron energy of $50 \mathrm{GeV}$ by means of superconducting cavities. A development programme of cavities made of $\mathrm{Nb}$ sheet was undertaken at CERN in 1979 and gave so far very encouraging results $1,2,3)$. Similar studies are also in progress in other laboratories. A recent review of these studies is given $i n^{4)}$. In parallel with this programme, a different approach has been pursued at CERN since 1980, which aims at producing Nbcoated copper cavities. This latter approach was expected to provide superior behaviour with respect to quenching. At high acceleration fields the performance of $\mathrm{Nb}$ sheet cavities is limited by resistive localized surface defects which heat up and may drive the neighbouring superconductor into the normal $8 \mathrm{tate}$ above a certain level of power dissipation. Increasing the thermal conductivity of the cavity wall material by replacing $\mathrm{Nb}$ with Cu should improve enormously the stability against quench by enhancing the critical power load 5$)$. This feature consequently should result in higher accelerating fields and/or insensitivity to the presence of resistive accidental spots as for instance they may be produced by dust particles. Further advantages of the Cu-Nb approach may consist in easier fabrication and lower cost of material. 


\section{Choice of the coating method}

Sputtering was preferred to other coating methods, as for instance chemical vapour deposition or evaporation, for many reasons. First, the low temperature during coating prevents crystal growth, annealing and deformation of the cavity. Second, uniformity of film thickness is guaranteed by proper cathode design. Third, the feasibility of this method is proven $6,7,8,9$ ) provided that UHV technology is used for which expertise is available at CERN. Another very attractive feature, so far not exploited, is that compounds with $T_{c}$ higher than $9.2 \mathrm{~K}$ may be deposited by sputtering. Since the surface resistance of a superconductor decreases exponentially with increasing $T_{c}$, these compounds offer much higher potential $Q$ values.

The major existing unknowns concerned the compatibility of $\mathrm{Cu}$ and $\mathrm{Nb}$ ( $\mathrm{Nb}$ is usually deposited on insulators) and the feasibility of coating onto the inside of an almost closed vessel, a feature which imposed the use of rotating cathodes. Mainly for this latter reason, diode sputtering was initially chosen.

\section{Experimental approach and results}

Owing to the large number of deposition parameters, the optimisation of the $\mathrm{Nb}$ films was carried out initially on small samples in a plane cathode configuration. Only upon reaching the desired quality level on samples, coating was extended to $3 \mathrm{GHz}$ cavities (semi-spherical shape with about $9 \mathrm{~cm}$ diameter) and subsequently to $500 \mathrm{MHz}$ cavities (also of semispherical shape, with a diameter of about $52 \mathrm{~cm})^{2}$. Coating of cavities was achieved by means of rotating cathodes shaped to provide a deposited film of uniform thickness. Information on these films was obtained from samples hanging at various points inside test cavities during coating. The vacuum systems used were all metal bakeable at above $200^{\circ} \mathrm{C}$, pumped by sputter-ion pumps and able to reach the $10^{-10}$ Torr range before coating. 


\subsection{Samples}

Samples initially obtained with diode sputtering never reached the $T_{C}$ of bulk Nb $(9.2 \mathrm{~K})$. Values obtained on quartz substrates were higher (above $8.7 \mathrm{~K}$ ) than on $\mathrm{Cu}$ (about $8.2 \mathrm{~K}$ ). The film surface was rough and its structure columnar. Auger depth analysis revealed the presence in the films of large amounts of $\mathrm{C}$ and $\mathrm{O}_{2}$, to which $\mathrm{Cu}$ added up in the case of $\mathrm{Cu}$ substrates. The films were brittle, of gray aspect and presented good adhesion to the substrate.

It is known from literature 10,11$)$ that both film purity and density are enhanced by bombardment of low energy ions (typically 60 to $80 \mathrm{eV}$ ) during film growth. This effect is usually obtained by applying to the substrate a negative bias (bias sputtering) with respect to the grounded walls of the vessel. Applying bias sputtering to the plane cathode configuration was straightforward and the improvement spectacular. Values of $T_{c}$ equal or even higher than those of bulk $\mathrm{Nb}$ were obtained. The impurity content was greatly reduced and limited to the uppermost layer only. No traces of $\mathrm{Cu}$ were observable in the film. The film surface was shining and its ductility and adhesion to the substrate good.

Applying bias sputtering to the cavities required introducing an electrode parallel to the cathode and biased positive with respect to the cavity envelope (for the $3 \mathrm{GHz}$ cavity) or applying the positive bias to the cathode shields for the $500 \mathrm{MHz}$ cavity. Samples obtained inside test cavities in this way were as good as those obtained in the plane configuration.

Optimisation of the discharge parameters in the three different configurations resulted approximately in the same values which are as follows. Cathode voltage $-1200 \pm 200 \mathrm{~V}$, bias voltage $80 \pm 10 \mathrm{~V}$, argon pressure about $5 \times 10^{-2}$ Torr. Films of thickness ranging from 1.5 to 4 jum were obtained in about $20 \mathrm{~h}$. 


\subsection{Cavities}

The performance of $3 \mathrm{GHz}$ cavities coated without bias electrode were poor. The best $Q$ value only reached $1.3 \times 10^{7}$. Adding the reference electrode biased at $+80 \mathrm{~V}$ with respect to the cavity enhanced the $Q$ value up to $1.2 \times 10^{8}$ at $4.2 \mathrm{~K}$ and low power. The maximum accelerating field was about $3.7 \mathrm{MV} \mathrm{m}^{-1}$.

More exciting were the results obtained on the $500 \mathrm{MHz}$ cavity, already at the first trial (with bias). At $4.2 \mathrm{~K}$ the following $Q$ values were measured. At very low field, $1.8 \times 10^{9}$; at $3 \mathrm{MVm}^{-1}, 1 \times 10^{9}$; at $5 \mathrm{MVm}^{-1}, 8$ $x 10^{8}$. Pushing the field above $6 \mathrm{MV}^{-1}$ produced an irreversible $Q$ deterioration, as indicated by the following values measured in a second run. At very low field about $10^{9}$; at $3 \mathrm{MVm}^{-1}, 7 \times 10^{8}$; at $5 \mathrm{MVm}^{-1}, 6 \times 10^{8}$; at $7 \mathrm{MVm}^{-1}, 5 \times 10^{8}$. At the maximum field which was reached $\left(8.6 \mathrm{MVm}^{-1}\right) \mathrm{Q}$ was about $3 \times 10^{8}$. At this stage no quench appeared, but the test was 1 imited by the large power dissipation and the characteristics of the power supply. With respect to the best cavities made of $\mathrm{Nb}$ sheet, the coated $500 \mathrm{MHz}$ cavity presented a higher maximum field but $Q$ values at equal fields about 2 times lower.

At distinction with samples, local film peel-off was experienced in both types of cavities, showing how critical is the cleaning step in the preparation process.

\section{Conclusions}

The available data confirm fully the benefits that copper was expected to offer in terms of stability against quench. Furthermore, evidence now exists that $\mathrm{Nb}$ films of reasonably good quality may be deposited on to the inside of $\mathrm{Cu}$ cavities of geonetry very close to that required for LEP (in this case the frequency will be $350 \mathrm{MHz}$ ). We are confident that the reasons of sporadic film peel-off or other kinds of local defects may be understood and cured. More of a problem may be to obtain reproducibly the high $Q$ values required for $L E P$ which are very close to the best values available with bulk Nb. Coating with a higher $\mathrm{T}_{c}$ material may be the solution to this problem. 


\section{Acknowledgements}

Uaing copper as cavity wall material was suggested by $P$. Bernard and E. Picasso. The authors are indebted to $M$. Wurgel for advice on cathode design, J. Genest for mechanical calculations and drawings, A. Boulmier and R. Kopp for surface cleaning, A. Grillot for Auger analysis, M. Blin for $T_{c}$ measurements, J-P. Bacher and J. Adam for electron microscope analysis,

$C$. Dalmas, J-M. Laurent and W. Weingarten for cavity measurements. X-ray diffraction measurements were carried out by $\emptyset$. Fischer's group at the Physics University of Geneva. Useful discussions with P. Bernard, H. Padamsee and W. Weingarten are gratefully acknowledged.

\section{References}

1. P. Bernard et al., Proc. of the lith Int. Conf. on High Energy Accelerators, p. 878, Geneva (1980).

2. P. Bernard et al., Proc. of the 12th Int. Conf. on High Energy Accelerators, Chicago (1983, to be published).

3. P. Bernard et al., CERN-EF/RF/83-6 (1983).

4. H. Piel, Proc. of the 12th Int. Conf. on High Energy Accelerators, Chicago (1983, to be published).

5. H. Padamsee, CERN-EF/RF/82-5 (1982).

6. S.A. Wolf, J.J. Kennedy, M. Nisenoff, J. Vac. Sci. Technol., 13, 1, p.145(1976).

7. M.J. Witcomb, Thin Solid Films, 74, P. 223 (1980).

8. Y. Saito, T. Anayama, J. Low Temp. Phys., 21, p. 169 (1975),

9. J. Edgecumbe, L.G. Rosner, D.E. Anderson, J. Appl. Phys., 35, No. 7, p. 2198 (1964).

10. K.L. Chopra, Thin Film Phenomena, p. 35, McGraw Hil1, New York, 1969.

11. J.J. Cuomo, J. Vac. Sci. Technol., 11 p. 34, (1974).

This note has been submitted for publication in Applied Physics Letters 